\title{
On Smoothness of the Green Function for the Complement of a Rarefied Cantor-Type Set
}

\author{
Muhammed Altun • Alexander Goncharov
}

Received: 24 April 2009 / Accepted: 18 August 2009 / Published online: 8 April 2010

(C) Springer Science+Business Media, LLC 2010

\begin{abstract}
Smoothness of the Green functions for the complement of rarefied Cantortype sets is described in terms of the function $\varphi(\delta)=\left(1 / \log \frac{1}{\delta}\right)$ that gives the logarithmic measure of sets. Markov's constants of the corresponding sets are evaluated.
\end{abstract}

Keywords Green's function · Markov's inequality · Cantor-type sets

Mathematics Subject Classification (2000) 31A15 • 41A10 • 41A17

\section{Introduction}

Let a compact set $K$ be regular with respect to the Dirichlet problem. Then the Green function $g_{\mathbb{C} \backslash K}$ of $\mathbb{C} \backslash K$ with pole at infinity is continuous throughout $\mathbb{C}$. Related to polynomial inequalities and some other applications, the problem of smoothness of $g_{\mathbb{C} \backslash K}$ near the boundary of $K$ has attracted the attention of many mathematicians (see, e.g., the survey [4] and the references given there). New incentive to analyze the problem has been provided by the monograph [17] by Totik, where the author characterized the smoothness of Green functions and harmonic measures in terms of the density $\Theta_{K}(t)$ (Theorems 2.1 and 2.2 in [17]). For the case $K \subset[0,1]$ with $0 \in K$, which we will consider in what follows, the density at 0 is measured by the function $\Theta_{K}(t)=m([0, t] \backslash K)$, where $m$ stands for the linear Lebesgue measure.

Communicated by Vilmos Totik.

M. Altun

Department of Mathematics, Adiyaman University, Adiyaman, Turkey

e-mail: maltun@adiyaman.edu.tr

A. Goncharov (凶)

Department of Mathematics, Bilkent University, Ankara, Turkey

e-mail: goncha@fen.bilkent.edu.tr 
The monotonicity of the Green function with respect to the set $K$ implies $g_{\mathbb{C} \backslash K}(z) \geq g_{\mathbb{C} \backslash[0,1]}(z)$ for $z \in \mathbb{C}$. In this way, we get the optimal behavior (Lip $\frac{1}{2}$ smoothness) near the origin of the function $g_{\mathbb{C} \backslash K}$ for $K \subset[0,1]$. Various conditions for optimal smoothness of $g_{\mathbb{C} \backslash K}$ in terms of metric properties of the set $K$ are suggested in $[9,17]$ and in papers of V. Andrievskii [2-4]. For example, the Green function corresponding to the classical Cantor set $K_{0}$ is Hölder continuous by [6], but is not optimal smooth, by Theorem 5.1 in [17]. A recent result on smoothness of $g_{\mathbb{C} \backslash K_{0}}$ can be found in [15].

Here we consider Cantor-type sets $K^{(\alpha)}$ with "lowest smoothness" of the corresponding Green function. Let $1<\alpha, 0<l_{1}<\frac{1}{2}$, and $2 l_{1}^{\alpha-1}<1$. Then $K^{(\alpha)}=$ $\bigcap_{s=0}^{\infty} E_{s}$, where $E_{0}=I_{1,0}=[0,1], E_{s}$ is a union of $2^{s}$ closed basic intervals $I_{j, s}$ of length $l_{s}=l_{s-1}^{\alpha}$, and $E_{s+1}$ is obtained by deleting the open concentric subinterval of length $h_{s}:=l_{s}-2 l_{s+1}$ from each $I_{j, s}$ with $j=1,2, \ldots, 2^{s}$. The set $K^{(\alpha)}$ is not polar if and only if $\alpha<2$ ([8, Chap. IV, Theorem 3]). Also, by Pleśniak [13], in the case of the Cantor type set, the corresponding set is regular if and only if it is not polar. Thus, in the case $1<\alpha<2$, the Green function $g_{\mathbb{C} \backslash K^{(\alpha)}}$ is continuous. We show that its modulus of continuity can be estimated in terms of the function $\varphi(\delta)=\left(1 / \log \frac{1}{\delta}\right)$, which is used in the definition of the logarithmic measure (see, e.g., [12, Chap. V, 6]). Here and subsequently, log denotes the natural logarithm.

Since $\Theta_{K^{(\alpha)}}(t)=t$, neither the estimation from Theorem 2.2 in [17] nor the previous general bound of Green functions given by Tsuji [18, Theorem III, 67] can be applied to our case. Let $\Pi_{n}$ denote the set of all polynomials of degree at most $n, \Pi=\bigcup_{n=0}^{\infty} \Pi_{n}$. Let $|f|_{K}:=\sup _{x \in K}|f(x)|$. We use the representation

$$
g_{\mathbb{C} \backslash K^{(\alpha)}}(z)=\sup \left\{\frac{\log |P(z)|}{\operatorname{deg} P}: P \in \Pi, \operatorname{deg} P \geq 1,|P|_{K^{(\alpha)}} \leq 1\right\},
$$

which follows on one hand from the Bernstein-Walsh lemma ([19, p. 77]) and on the other hand by the possibility of approximating $\exp g_{\mathbb{C} \backslash K^{(\alpha)}}(z)$, for example by the sequence $\left(\left|\Phi_{n}(z)\right|^{1 / n}\right)$, where $\Phi_{n}$ denotes the normalized Fekete polynomial (see, e.g., [14, Theorem 11.1]).

There is a strong connection between the smoothness of $g_{\mathbb{C} \backslash K}$ near the boundary of $K$ and values of Markov's factors $M_{n}(K)=\sup _{P \in \Pi_{n}} \frac{\left|P^{\prime}\right|_{K}}{|P|_{K}}$, which are well defined for any infinite set $K$. Indeed, suppose that for some increasing continuous function $F$ we have $g_{\mathbb{C} \backslash K}(z) \leq F(\delta)$ for $\operatorname{dist}(z, K) \leq \delta$. Then for any $P \in \Pi_{n}$ the BernsteinWalsh inequality gives $|P(z)| \leq|P|_{K} \exp [n \cdot F(\delta)]$. Applying Cauchy's formula for $P^{\prime}$ on the circle with center at $\zeta \in K$ and of radius $\delta$ yields $\left|P^{\prime}(\zeta)\right| \leq \delta^{-1} \exp [n$. $F(\delta)]|P|_{K}$. This gives the bound $M_{n}(K) \leq \inf _{\delta} \delta^{-1} \exp [n \cdot F(\delta)]$. Particularly, if we choose $\delta$ with $F(\delta)=n^{-1}$, then $M_{n}(K) \leq e \cdot\left[F_{-1}\left(n^{-1}\right)\right]^{-1}$, where $F_{-1}$ stands for the inverse to $F$ function. For example, the Hölder continuity of the Green function $g_{\mathbb{C} \backslash K}$ implies Markov's property of the set $K$, which means that there are constants $C, r$ such that $M_{n}(K) \leq C n^{r}$ for all $n$.

Here we give an asymptotic for $M_{n}\left(K^{(\alpha)}\right)$ which is new compared to the previous results about Markov's constants of Cantor-type sets (see [10, Example 7], [16], and [7]). 
As a method we employ local interpolations of functions that were used in [1] to present extension operators for the Whitney spaces $\mathcal{E}\left(K^{(\alpha)}\right)$ and in [11] to construct topological bases in spaces $\mathcal{E}(K)$ for more general Cantor-type sets.

\section{Results}

Given $1<\alpha<2$, let $K^{(\alpha)}$ be the Cantor set defined in the introduction, $\varphi(\delta)=$ $\left(\log \frac{1}{\delta}\right)^{-1}$ for $0<\delta<1$ and $\gamma=\log \frac{2}{\alpha} / \log \alpha$.

Theorem 1 For every $0<\varepsilon<\gamma$ there exist constants $\delta_{0}, C_{0}$, depending on $\alpha$ and $\varepsilon$, such that $g_{\mathbb{C} \backslash K^{(\alpha)}}(z) \leq C_{0} \varphi^{\gamma-\varepsilon}(\delta)$ for $z \in \mathbb{C}$ with $\operatorname{dist}\left(z, K^{(\alpha)}\right)=\delta \leq \delta_{0}$.

Theorem 2 There are constants $\delta_{0}, \varepsilon_{0}$, depending only on $\alpha$, such that $g_{\mathbb{C} \backslash K^{(\alpha)}}(-\delta)$ $\geq \varepsilon_{0} \varphi^{\gamma}(\delta)$ for $\delta \leq \delta_{0}$.

Corollary 1 If $1<\alpha<2$, then for every $0<\varepsilon<\gamma$ there exists a constant $C_{1}$ such that $M_{n}\left(K^{(\alpha)}\right) \leq \exp \left[C_{1} \cdot n^{(1+\varepsilon) \frac{\log \alpha}{\log 2}}\right]$ for $n \in \mathbb{N}$. On the other hand, for each $\alpha>1$ we have $M_{n}\left(K^{(\alpha)}\right)>\exp \left[\alpha^{-2} \cdot n^{\frac{\log \alpha}{\log 2}}\right]$ for $n \in \mathbb{N}$.

\section{Proof of Theorem 2}

Let us first prove the more simple sharpness result.

Without loss of generality we can suppose that $l_{1}=e^{-1}$, so $l_{s}=\exp \left(-\alpha^{s-1}\right)$. If $l_{q+1}<\delta \leq l_{q}$, then $\alpha^{-q}<\varphi(\delta) \leq \alpha^{-q+1}$. Since $\alpha^{-\gamma}=\alpha / 2$, we have

$$
\left(\frac{\alpha}{2}\right)^{q}<\varphi^{\gamma}(\delta) \leq\left(\frac{\alpha}{2}\right)^{q-1}
$$

Let us fix $q_{0}$ with $(\alpha / 2)^{q_{0}-1} \leq(\alpha-1) / 2, \delta_{0}=l_{q_{0}}$, and $\varepsilon_{0}=\frac{\alpha}{8} \frac{\alpha-1}{2-\alpha}$. In view of (1) and (2), it is enough for given $l_{q+1}<\delta \leq l_{q} \leq l_{q_{0}}$ to find a polynomial $P \in \Pi_{n}$ with $|P|_{K^{(\alpha)}} \leq 1$ such that

$$
\frac{\log |P(-\delta)|}{n} \geq \frac{1}{4} \frac{\alpha-1}{2-\alpha}\left(\frac{\alpha}{2}\right)^{q} .
$$

For fixed $m \in \mathbb{N}$ let $\left(x_{k}\right)_{k=1}^{2^{m}}$ be the set of all endpoints of the basic intervals $I_{j, m-1}$ with $j=1,2, \ldots, 2^{m-1}$. We arrange them in increasing order, so $x_{1}=0, x_{2}=$ $l_{m-1}, x_{3}=l_{m-2}-l_{m-1}, \ldots, x_{2^{k}}=l_{m-k}, \ldots, x_{2^{m}}=1$. Set $\omega(z)=\prod_{k=1}^{2^{m}}\left(z-x_{k}\right)$. Then the fundamental Lagrange polynomial $L_{1}(z)=\left(\omega(z) / z \cdot \omega^{\prime}(0)\right)$ has the norm $\left|L_{1}\right|_{K^{(\alpha)}}$ equal 1 , as is easy to check. Indeed, if $x \in K^{(\alpha)} \cap I_{1, m-1}$, then $\left|L_{1}(x)\right| \leq$ $\left|L_{1}(0)\right|=1$, by the monotonicity of $\omega(z) / z$ there. Otherwise, $x \in K^{(\alpha)} \cap I_{j, m-1}$ with $j=2, \ldots, 2^{m-1}$, and $|\omega(x)| \leq l_{m} \cdot x_{2} \cdot x_{3} \cdots x_{2^{m}}$, so $\left|L_{1}(x)\right| \leq l_{m} / x<1$. 
Now for given $q$ we take $m=2 q, n=4^{q}-1$ and $P=L_{1} \in \Pi_{n}$. Then

$$
|P(-\delta)|=\prod_{k=2}^{2^{q-1}} \frac{x_{k}+\delta}{x_{k}} \prod_{k=2^{q-1}+1}^{4^{q}}\left(1+\frac{\delta}{x_{k}}\right) .
$$

We disregard the second product, which exceeds 1 , and $x_{k}$ in the numerator of the first product. For its denominator we have $\prod_{k=2}^{2^{q-1}} x_{k}<l_{2 q-1} \cdot l_{2 q-2}^{2} \cdots l_{q+1}^{2^{q-2}}=l_{q+1}^{\varkappa}$, where $\varkappa=2^{q-2}+\alpha \cdot 2^{q-3}+\cdots+\alpha^{q-3} \cdot 2+\alpha^{q-2}=(2-\alpha)^{-1}\left(2^{q-1}-\alpha^{q-1}\right)$. Therefore, $|P(-\delta)|>l_{q+1}^{2^{q-1}-1-\varkappa}=\exp \left[\left(\varkappa-2^{q-1}+1\right) \alpha^{q}\right]$. Here, $\varkappa-2^{q-1}=2^{q-1}\left[\frac{\alpha-1}{2-\alpha}-\right.$ $\left.\frac{1}{2-\alpha}\left(\frac{\alpha}{2}\right)^{q-1}\right]>2^{q-2} \frac{\alpha-1}{2-\alpha}$, due to the choice of $q_{0}$. Thus, $\log |P(-\delta)|>2^{q-2} \alpha^{q} \frac{\alpha-1}{2-\alpha}$. This gives the desired bound (3), since $n<4^{q}$.

\section{Proof of Theorem 1}

Let us fix $\varepsilon$ with $0<\varepsilon<\gamma$. As above, we suppose that $l_{1}=e^{-1}$.

We want to find $q_{0}$ and $C_{0}$ such that if $\operatorname{dist}\left(z, K^{(\alpha)}\right)=\delta \in\left(l_{q+1}, l_{q}\right]$ with $q \geq q_{0}$, then

$$
g_{\mathbb{C} \backslash K^{(\alpha)}}(z) \leq C_{0}\left(\frac{\alpha}{2}\right)^{q} \alpha^{q \varepsilon} .
$$

We set $Q_{\alpha}:=\frac{\alpha}{\alpha-1} \log \frac{2}{\alpha}$ and choose $q_{0}$ so large that for $q \geq q_{0}$ the following conditions hold:

$$
\begin{aligned}
Q_{\alpha} q & <\alpha^{q-1}, \\
\log \left(Q_{\alpha} q\right) & <q \varepsilon \log ^{2} \alpha, \\
\left(\frac{\alpha}{2}\right)^{q} & <\frac{1}{4} .
\end{aligned}
$$

Now for fixed $q \geq q_{0}$ we take $k=\left[\frac{\log \left(Q_{\alpha} q\right)}{\log \alpha}\right]+1$, where $[x]$ denotes the greatest integer in $x$. Due to the choice of $Q_{\alpha}$, we have

$$
l_{k}^{\alpha-1}<\left(\frac{\alpha}{2}\right)^{q} \leq l_{k-1}^{\alpha-1},
$$

that is, $k=\min \left\{j: l_{j}^{\alpha-1}<\left(\frac{\alpha}{2}\right)^{q}\right\}$. Since (5) is equivalent to $l_{q-1}^{\alpha-1}<\left(\frac{\alpha}{2}\right)^{q}$, we get $k<q$. Also (6) implies

$$
2^{k}<2 \alpha^{q \varepsilon}
$$

as is easy to check.

Arguing as in the proof of Theorem 2.2 in [17], we see that it is enough to consider (4) only for $z=-\delta$. Let us fix any polynomial $P$ with $|P|_{K^{(\alpha)}} \leq 1$. Let $m \in \mathbb{N}$ be such that $2^{m-1} \leq \operatorname{deg} P<2^{m}$. In view of (1), we can reduce (4) to

$$
\log |P(-\delta)| \leq C_{0} 2^{m-q} \alpha^{q(1+\varepsilon)},
$$


and what is more, since polynomials in the representation (1) can be of arbitrary large degree, we can suppose without loss of generality that $m \geq 2 q$.

We interpolate $P$ on the interval $I_{1, k}$ at $2^{m}$ endpoints of $I_{j, k+m-1}$ with $j=$ $1,2, \ldots, 2^{m-1}$. Thus, $x_{1}=0, x_{2}=l_{k+m-1}, x_{3}=l_{k+m-2}-l_{k+m-1}, \ldots, x_{2^{i}}=l_{k+m-i}$, $\ldots, x_{2^{m}}=l_{k}$. Here $\omega(z)=\prod_{i=1}^{2^{m}}\left(z-x_{i}\right)$ and $L_{j}(z)=\frac{\omega(z)}{\left(z-x_{j}\right) \cdot \omega^{\prime}\left(x_{j}\right)}$ for $1 \leq j \leq 2^{m}$. Since $\operatorname{deg} P<2^{m}$, the interpolating polynomial $\mathcal{L}_{2^{m}-1}=\sum_{j=1}^{2^{m}} P\left(x_{j}\right) L_{j}$ coincides with $P$. In our case $\left|P\left(x_{j}\right)\right| \leq 1$. Therefore,

$$
|P(-\delta)| \leq \sum_{j=1}^{2^{m}}\left|L_{j}(-\delta)\right|
$$

Let us fix any $1 \leq j \leq 2^{m}$ and estimate $\left|L_{j}(-\delta)\right|$ from above. We have $|\omega(-\delta)|<l_{q} \cdot\left(l_{q}+l_{k+m-1}\right) \cdot\left(l_{q}+l_{k+m-2}\right)^{2} \cdots\left(l_{q}+l_{k}\right)^{2^{m-1}}=l_{q}^{2^{k+m-q}} \cdot 2^{2^{k+m-q-1}} \cdot$ $l_{q-1}^{2^{k+m-q}} \cdots l_{k}^{2^{m-1}} \cdot B$, where $B=\left(1+\frac{l_{k+m-1}}{l_{q}}\right)\left(1+\frac{l_{k+m-2}}{l_{q}}\right)^{2} \cdots\left(1+\frac{l_{q+1}}{l_{q}}\right)^{2^{k+m-q-2}}(1+$ $\left.\frac{l_{q}}{l_{q-1}}\right)^{2^{k+m-q}} \cdots\left(1+\frac{l_{q}}{l_{k}}\right)^{2^{m-1}}$. On the other hand, by the structure of the set $K^{(\alpha)}$, $\left|\omega^{\prime}\left(x_{j}\right)\right|>l_{k+m-1} \cdot h_{k+m-2}^{2} \cdot h_{k+m-3}^{4} \cdots h_{k}^{2^{m-1}}=l_{k+m-1} \cdot l_{k+m-2}^{2} \cdots l_{k}^{2^{m-1}} \cdot \beta$ with $\beta=\left(1-2 \frac{l_{k+m-1}}{l_{k+m-2}}\right)^{2} \cdots\left(1-2 \frac{l_{k+1}}{l_{k}}\right)^{2^{m-1}}$. Also, $\left|-\delta-x_{j}\right| \geq l_{q+1}$. Therefore,

$$
\left|L_{j}(-\delta)\right| \leq \frac{B}{\beta} 2^{2^{k+m-q-1}} l_{q}^{\varkappa}
$$

with $\varkappa=2^{k+m-q-1}-\alpha-\left[\alpha^{k+m-q-1}+2 \alpha^{k+m-q-2}+\cdots+2^{k+m-q-2} \alpha\right]=$ $2^{k+m-q-1}-\alpha-2^{k+m-q-1} \cdot \frac{\alpha}{2-\alpha}+\frac{\alpha^{k+m-q}}{2-\alpha}=-2^{k+m-q} \cdot \frac{\alpha-1}{2-\alpha}+\frac{\alpha^{k+m-q}}{2-\alpha}-\alpha$.

From this, $|P(-\delta)| \leq 2^{m} \frac{B}{\beta} 2^{2^{k+m-q-1}} l_{q}^{\varkappa}$, and the desired inequality (10) is analogous to

$$
m \log 2+\log B-\log \beta+2^{k+m-q-1} \log 2+\varkappa \log l_{q} \leq C_{0} 2^{m-q} \alpha^{q(1+\varepsilon)} .
$$

Here, $\varkappa \log l_{q}=2^{k+m-q} \cdot \alpha^{q-1} \cdot \frac{\alpha-1}{2-\alpha}-\frac{\alpha^{k+m-1}}{2-\alpha}+\alpha^{q}$. Since $k+m>2 q$, the sum of the last two terms is negative. We neglect this sum. Thus it is enough to show

$$
m+2^{k+m-q-1}+\log B-\log \beta+2^{k+m-q} \cdot \alpha^{q-1} \cdot \frac{\alpha-1}{2-\alpha} \leq C_{0} 2^{m-q} \alpha^{q(1+\varepsilon)} .
$$

Each of the 5 summands on the left will be estimated separately from above by $R:=2^{m-q} \alpha^{q(1+\varepsilon)}$.

$\mathbf{S}_{\mathbf{1}}:=m$. Since $m \geq 2 q$, we have $2^{m-q} \geq 2^{m / 2} \geq m / 2$, so $S_{1} \leq 2 R$.

$\mathbf{S}_{\mathbf{2}}:=2^{k+m-q-1}<\alpha^{q \varepsilon} 2^{m-q}$, by (9). Thus, $S_{2}<R$.

$\mathbf{S}_{\mathbf{3}}:=\log B$. Clearly, $l_{q} / l_{q-1}>l_{q+1} / l_{q}$, since $l_{q-1}^{(\alpha-1)^{2}}<1$. Therefore, $B<(1+$ $\left.\frac{l_{q}}{l_{q-1}}\right)^{2^{m}}$ and $\log B<2^{m} l_{q-1}^{\alpha-1}<2^{m}\left(\frac{\alpha}{2}\right)^{q}$, by (5). Therefore, $S_{3}<R$.

$\mathbf{S}_{\mathbf{4}}:=-\log \beta$. Here, $\frac{l_{k+1}}{l_{k}}>\frac{l_{j+1}}{l_{j}}$ for $j=k+1, \ldots, k+m-2$. Consequently, $\beta>\left(1-2 \frac{l_{k+1}}{l_{k}}\right)^{2^{m}}$ and $\log \beta>-2^{m+2} l_{k}^{\alpha-1}$. Here we use the inequality $\log (1-x)>$ 
$-2 x$, which is valid for $0<x<1 / 2$. In our case $x=2 l_{k}^{\alpha-1}<1 / 2$, by (8) and (7). Additionally, (8) implies $S_{4}<2^{m+2}\left(\frac{\alpha}{2}\right)^{q}<4 R$.

Finally, $\mathbf{S}_{\mathbf{5}}:=2^{k+m-q} \cdot \alpha^{q-1} \cdot \frac{\alpha-1}{2-\alpha}<\frac{2}{\alpha} \cdot \frac{\alpha-1}{2-\alpha} \cdot R$, by (9). This gives (11) with $C_{0}=8+\frac{2}{\alpha} \cdot \frac{\alpha-1}{2-\alpha}$ and completes the proof of Theorem 1 .

\section{Markov's Factors}

By the arguments given in the introduction,

$$
M_{n}\left(K^{(\alpha)}\right) \leq \inf _{\delta \leq \delta_{0}} \delta^{-1} \exp \left[n \cdot C_{0} \varphi^{\gamma-\varepsilon}(\delta)\right] .
$$

Let us take $\delta=\exp \left(-n^{\frac{\log \alpha}{\log 2}}\right)$. Then $\varphi(\delta)=n^{-\frac{\log \alpha}{\log 2}}$ and $n \cdot \varphi^{\gamma}(\delta)=n^{\frac{\log \alpha}{\log 2}}$. Therefore, $M_{n}\left(K^{(\alpha)}\right) \leq \exp \left[\left(C_{0}+1\right) \cdot n^{(1+\varepsilon) \frac{\log \alpha}{\log 2}}\right]$ for large enough $n$. By increasing the constant, if necessary, we have the first bound in corollary.

Of course these arguments cannot be used for the case of polar sets $K^{(\alpha)}$ with $\alpha \geq 2$. But the lower bound of $M_{n}\left(K^{(\alpha)}\right)$ can be presented easily for any $\alpha>1$. Indeed, let us fix $n \in \mathbb{N}$. Let $2^{m} \leq n<2^{m+1}$. We take the same $2^{m}$ points $\left(x_{k}\right)_{k=1}^{2^{m}}$ as in Sect. 3 and $P(z)=\prod_{k=1}^{2^{m}}\left(z-x_{k}\right)$. Then $|P|_{K^{(\alpha)}}=\left|P\left(l_{m}\right)\right|=l_{m}\left(x_{2}-\right.$ $\left.l_{m}\right) \cdots\left(1-l_{m}\right)<l_{m} \cdot \prod_{k=2}^{2^{m}} x_{k}$. On the other hand, $\left|P^{\prime}(0)\right|=\prod_{k=2}^{2^{m}} x_{k}$. By definition, the sequence $\left(M_{n}\right)$ is not decreasing. Therefore, $M_{n}\left(K^{(\alpha)}\right) \geq M_{2^{m}}\left(K^{(\alpha)}\right) \geq$ $\left|P^{\prime}(0)\right| /|P|_{K^{(\alpha)}}>l_{m}^{-1}=\exp \alpha^{m-1}=\exp \left[\alpha^{-2} \cdot\left(2^{m+1}\right)^{\frac{\log \alpha}{\log 2}}\right]>\exp \left(\alpha^{-2} \cdot n^{\frac{\log \alpha}{\log 2}}\right)$.

\section{Remarks}

1. The function $\varphi(\delta)=\left(1 / \log \frac{1}{\delta}\right)$ was used in [5] to define the logarithmic dimension of compact sets, as the Hausdorff dimension corresponding to the function $\varphi$. In particular, the logarithmic dimension of $K^{(\alpha)}$ is $\frac{\log 2}{\log \alpha}$.

2. We conjecture that the genuine modulus of continuity of $g_{\mathbb{C} \backslash K^{(\alpha)}}(z)$ is given by $\varphi^{\gamma}\left(\operatorname{dist}\left(z, K^{(\alpha)}\right)\right)$, that is, $-\varepsilon$ in the upper bound can be removed by another distribution of interpolating nodes, which will be closer to the distribution of the Fekete points on the set $K^{(\alpha)} \cap I_{1, k}$. This will mean that $\exp \left(\alpha^{-2} \cdot n^{\frac{\log \alpha}{\log 2}}\right)<M_{n}\left(K^{(\alpha)}\right)<$ $\exp \left(C \cdot n^{\frac{\log \alpha}{\log 2}}\right)$ for some constant $C$.

Acknowledgement The authors wish to thank the referee for valuable criticism and for suggestions to shorten the paper.

\section{References}

1. Altun, M., Goncharov, A.: A local version of the Pawłucki-Pleśniak extension operator. J. Approx. Theory 132, 34-41 (2005)

2. Andrievskii, V.V.: The highest smoothness of the Green function implies the highest density of a set. Ark. Mat. 42, 217-238 (2004) 
3. Andrievskii, V.V.: On optimal smoothness of the Green function for the complement of a Cantor-type set. Constr. Approx. 24, 113-122 (2006)

4. Andrievskii, V.V.: Constructive function theory on sets of the complex plane through potential theory and geometric function theory. Surv. Approx. Theory 2, 1-52 (2006)

5. Arslan, B., Goncharov, A., Kocatepe, M.: Spaces of Whitney functions on Cantor-type sets. Can. J. Math. 54(2), 225-238 (2002)

6. Białas, L., Volberg, A.: Markov's property of the Cantor ternary set. Stud. Math. 104, 259-268 (1993)

7. Białas-Cieź, L., Eggink, R.: L-regularity of Markov sets and of m-perfect sets in the complex plane. Constr. Approx. 27, 237-252 (2008)

8. Carleson, L.: Selected Problems on Exceptional Sets. Princeton Univ. Press, Princeton (1967)

9. Carleson, L., Totik, V.: Hölder continuity of Green's functions. Acta Sci. Math. (Szeged) 70(3-4), 557-608 (2004)

10. Erdélyi, T., Kroó, A., Szabados, J.: Markov-Bernstein type inequalities on compact subsets of $R$. Anal. Math. 26(1), 17-34 (2000)

11. Goncharov, A.: Basis in the spaces of $C^{\infty}$-functions on Cantor-type sets. Constr. Approx. 23(3), 351-360 (2006)

12. Nevanlinna, R.: Analytic Functions. Springer, Berlin (1970)

13. Pleśniak, W.: A Cantor regular set which does not have Markov's property. Ann. Pol. Math. 51, 269274 (1990)

14. Pommerenke, C.: Univalent Functions. Vandenhoeck and Ruprecht, Göttingen (1975)

15. Ransford, T., Rostand, J.: Hölder exponents of Green's functions of Cantor sets. Comput. Methods Funct. Theory 1, 151-158 (2008)

16. Totik, V.: Markoff constants for Cantor sets. Acta Sci. Math. (Szeged) 60, 715-734 (1995)

17. Totik, V.: Metric Properties of Harmonic Measures. Mem. Am. Math. Soc. 184, 867 (2006)

18. Tsuji, M.: Potential Theory in Modern Function Theory. Maruzen, Tokio (1959)

19. Walsh, J.L.: Interpolation and Approximation by Rational Functions in the Complex Domain. Colloquium Publications, vol. 20. Am. Math. Soc., Providence (1960) 